\title{
Uma proposta de solução para o Aircraft Recovery Problem de companhias aéreas regulares de pequeno porte
}

\author{
Glend Kleiser Gouveia Dias ${ }^{1}$, Anand Subramanian² e Lucídio dos Anjos Formiga Cabral ${ }^{3}$
}

\begin{abstract}
Resumo: A resolução do Aircraft Recovery Problem consiste em redefinir o sequenciamento das aeronaves, após interrupções das operações, que implique no menor custo operacional às companhias aéreas de voos regulares. O método adotado neste trabalho baseou-se na construção de uma rede tempo-espaço a partir dos dados de pousos e decolagens de uma companhia aérea brasileira. O modelo matemático do fluxo de custo mínimo foi implementado para o problema, o qual foi resolvido via Programação Linear Inteira. Foram realizados testes computacionais com centenas de instâncias e os resultados obtidos sugerem que a abordagem proposta é capaz de encontrar soluções melhores quando comparados com a simples estratégia de cancelar os voos atribuídos às aeronaves indisponíveis. Também foram realizados testes adicionais para verificar o impacto do fornecimento de uma aeronave extra no aeroporto com o maior número de voos operados pela companhia aérea estudada.

Palavras-chave: gestão das interrupções, aviação civil, Aircraft Recovery Problem, otimização.
\end{abstract}

Abstract: The Aircraft Recovery Problem consists of redefining the sequencing of aircrafts, after interruptions of operations,
that minimizes the operational costs of airlines with regular flights. The method used in this work is based on a space-time
network that is built after collecting real-world data associated with landings and takeoffs of a Brazilian airline company. A
mininum cost flow mathematical model was implemented for the problem, which was solved by means of a linear integer
programming solver. Computational experiments were carried out over hundreds of instances and the results obtained suggest
that the proposed approach is capable of finding better solutions when compared to the straightforward strategy of cancelling
the flights assigned to unavailable aircrafts. Additional tests were also performed to verify the impact of providing an extra
aircraft in the airport with the largest number of flights operated by the airline studied. Keywords: disruption management, civil aviation, Aircraft Recovery Problem, optimization.

\section{INTRODUÇÃO}

A lei 7.565, conhecida como Código Brasileiro de Aeronáutica (CBA), estabelece que o sistema de aviação civil brasileiro é composto basicamente por: transporte aéreo, indústria aeronáutica, infraestrutura aeroportuária e espaço aéreo (BRASIL, 1986a).

Os operadores regulares estão inseridos dentro do transporte aéreo e são aqueles que possuem Horário de Transporte (HOTRAN) definido, ou seja, já existe uma prévia aprovação da Agência Nacional de Aviação Civil (ANAC) que formaliza as concessões para a exploração de linhas aéreas internacionais e domésticas de passageiros pelas companhias aéreas, com os respectivos horários, números de voos, frequências, tipos de aeronaves e assentos disponíveis (BRASIL, 2000b).

Companhias aéreas como GOL, TAM, AVIANCA, AZUL, possuem elevados custos operacionais com combustível, manutenção, arrendamento e seguro das aeronaves, salário dos tripulantes, etc (ANAC, 2013). Outros custos indesejáveis estão relacionados aos atrasos e/ou cancelamentos dos voos devido à problemas mecânicos, mau tempo, congestionamento de aeroportos, problemas de segurança, etc.

Como forma de mitigar esses custos adicionais, Kohl et al. (2007) apontam os 3 objetivos básicos da gestão das

\footnotetext{
1 Glend Kleiser Gouveia Dias, Departamento de Engenharia de Produção, UFPB. (glend.dias@hotmail.com)

2 Anand Subramanian, Departamento de Sistemas de Computação, UFPB. (anandsubraman@hotmail.com)

${ }^{3}$ Lucídio dos Anjos Formiga Cabral, Departamento de Computação Científica, UFPB. (lucidiocabral@gmail.com)
}

Manuscrito recebido em 17/10/2016 e aprovado para publicação em 29/11/2016.

Este artigo é parte de TRANSPORTES v. 25, n. 1, 2017. ISSN: 2237-1346 (online). DOI:10.14295/transportes.v25i1.1265 interrupções: (a) Cumprir o contrato de transporte firmado entre a companhia aérea e o passageiro, (b) Reduzir os custos operacionais ocasionados pelas interrupções e (c) Possibilitar a retomada das operações normais o mais rápido possível.

Dentro da gestão das interrupções, diversos autores têm estudado a solução do Airline Recovery Problem, composto basicamente por 3 subproblemas: Aircraft Recovery Problem (ARP), Crew Recovery Problem (CRP) e Passenger Recovery Problem (PRP) (BELOBABA et al, 2009). Esses subproblemas podem ser tratados isoladamente ou integrados.

A resolução de cada um dos subproblemas tem como finalidades possibilitar o cumprimento dos voos planejados com o menor número de atrasos e cancelamentos, incidir o menor custo para a companhia aérea e retomar as operações normais o mais rápido possível. O ARP trata do insumo aeronave, o CRP da reprogramação da escala dos tripulantes e o PRP da acomodação e assistência aos passageiros.

Os primeiros trabalhos sobre a recuperação aérea foram desenvolvidos por Teodorovic e Guberinic (1984) com ênfase no problema de recuperação de aeronaves. Para esses autores, o replanejamento otimizado dos voos após um determinado período de indisponibilidade das aeronaves de uma companhia aérea pode minimizar os atrasos dos passageiros.

Para Bard et al. (2001), o problema do ARP pode ser tratado a partir da construção de uma rede tempo-espaço com bandas de tempo como uma variação do problema de fluxo de custo mínimo e resolução do problema via Programação Linear Inteira (PLI).

O seu trabalho é complementar ao de Arguello et al. (1997a), tendo em vista que este emprega a metaheurística GRASP para a resolução do ARP em grandes companhias aéreas. 
Para a situação que integra simultaneamente a recuperação de aeronaves e passageiros, Bisaillon et al. (2011) empregaram uma Large Neighbourhood Search para resolver o problema e testaram em 2009 o algoritmo em 18 instâncias dentro da competição conhecida como ROADEF Challenge, obtendo a primeira colocação. Já o estudo de Nickkar et al. (2015) baseou-se na resolução do ARP e PRP a partir do método de decomposição Dantzig-Wolfe e heurística langragiana.

Para a proposição de solução do ARP, discute-se o seguinte problema: a partir da programação original de voos previstos no HOTRAN de uma companhia aérea brasileira, e após a ocorrência de indisponibilidades de aeronaves, quais os novos sequenciamentos das aeronaves restantes permitirão o cumprimento dos voos programados com o menor custo?

Devido às diversas possibilidades de ocorrência de interrupções das operações que causam impactos econômicos negativos, sobretudo em períodos de alta demanda por passageiros, e também pela necessidade de as companhias aéreas possuírem metodologias eficientes que minimizem os custos operacionais nessas situações, existe um notório interesse no desenvolvimento de ferramentas de resposta rápida e eficaz de apoio à tomada de decisões nos momentos de contingenciamento.

O objetivo geral deste estudo consistiu na proposição de uma solução para o ARP de companhias aéreas regulares de pequeno porte. Os objetos intermediários foram alcançados a partir da definição do modelo matemático, descrição detalhada do método de resolução do problema, bem como a implementação em linguagem computacional da técnica e realização de testes a partir da criação de instâncias.

Na seção Introdução foi apresentada uma breve revisão da literatura, problema e objetivos do estudo. Na Formulação Matemática é discutido o modelo matemático adotado e nos Aspectos Metodológicos as etapas para a resolução do ARP. Por fim, na seção Resultados discute-se sobre a eficácia do método empregado e na Conclusão são apresentados os aspectos contributivos do estudo e as perspectivas para pesquisas futuras.

\section{FORMULAÇÃO MATEMÁTICA}

Antes da apresentação do modelo matemático, é importante a discussão dos conceitos básicos sobre o problema de fluxo de custo mínimo e as principais definições que o envolvem o ARP.
Seja $G=(M, A)$ uma rede orientada por um conjunto $M$ de $n$ nós e o conjunto $A$ de $m$ arcos orientados. Cada arco $(i, j) \in A$ tem um custo associado $c_{i j}$ que representa o custo por unidade de fluxo naquele arco e tem associado uma capacidade mínima de fluxo $L_{i j}$ através de $(i, j)$ e capacidade máxima de fluxo $U_{i j}$ através de $(i, j)$. O valor $b_{i}$ representa o suprimento/demanda dos nós $i$ e o valor $x_{i j} \mathrm{o}$ fluxo entre os nós $i$ e $j$ (TAHA, 2008).

\section{Função Objetivo:}

$$
\text { Minimizar } \sum_{i \in M} \sum_{j \in M} c_{i j} x_{i j}
$$

Sujeito a:

$$
\begin{gathered}
\sum_{j \in M} x_{i j}-\sum_{k \in M} x_{k i}=b_{i}, \quad \forall i \in M \\
L_{i j} \leq x_{i j} \leq U_{i j}
\end{gathered}
$$

A função objetivo (1) busca minimizar o custo total da rede. As restrições (2) satisfazem as exigências de cada nó quanto ao fluxo de entrada e saída, enquanto as restrições (3) impõem os limites inferior e superior de fluxo através dos arcos.

Como exemplo dessa definição, uma companhia aérea necessita transportar mercadorias dos nós 1 e 2 para os nós 5, 6 e 7, conforme apresentado na Figura 1, mas não possui voos diretos entre os nós de origem e os nós de destino e necessariamente precisa passar pelos nós 3 e 4 . Os números externos aos nós representam o suprimento/demanda em toneladas e os números nos arcos representam os custos por unidade de tonelada transportada.

Neste problema o interesse é determinar o melhor caminho para transportar as mercadorias entre os nós de suprimento e os nós de demanda com o menor custo total. Existe a limitação que no nó 4 só entra ou sai no máximo 50 toneladas em mercadorias. Resolvendo o problema, a solução encontrada é aquela que acarreta um custo de $\$ 1250$.

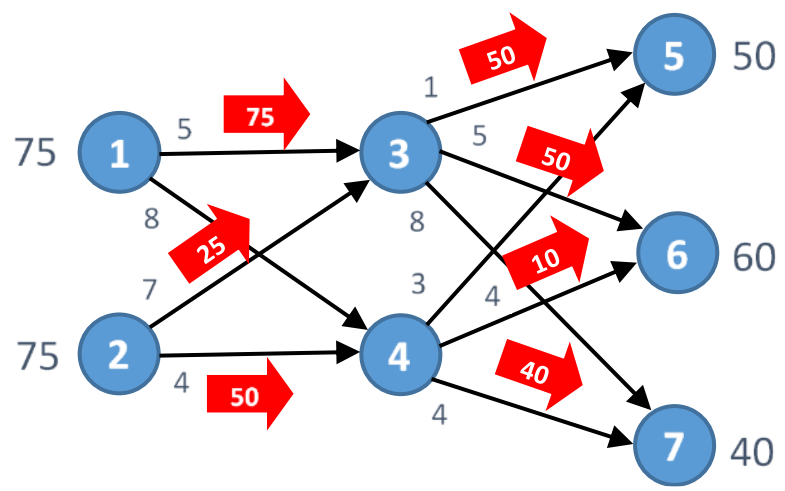

Figura 1. Solução para o fluxo de custo mínimo Fonte: Bazargan (2012) 


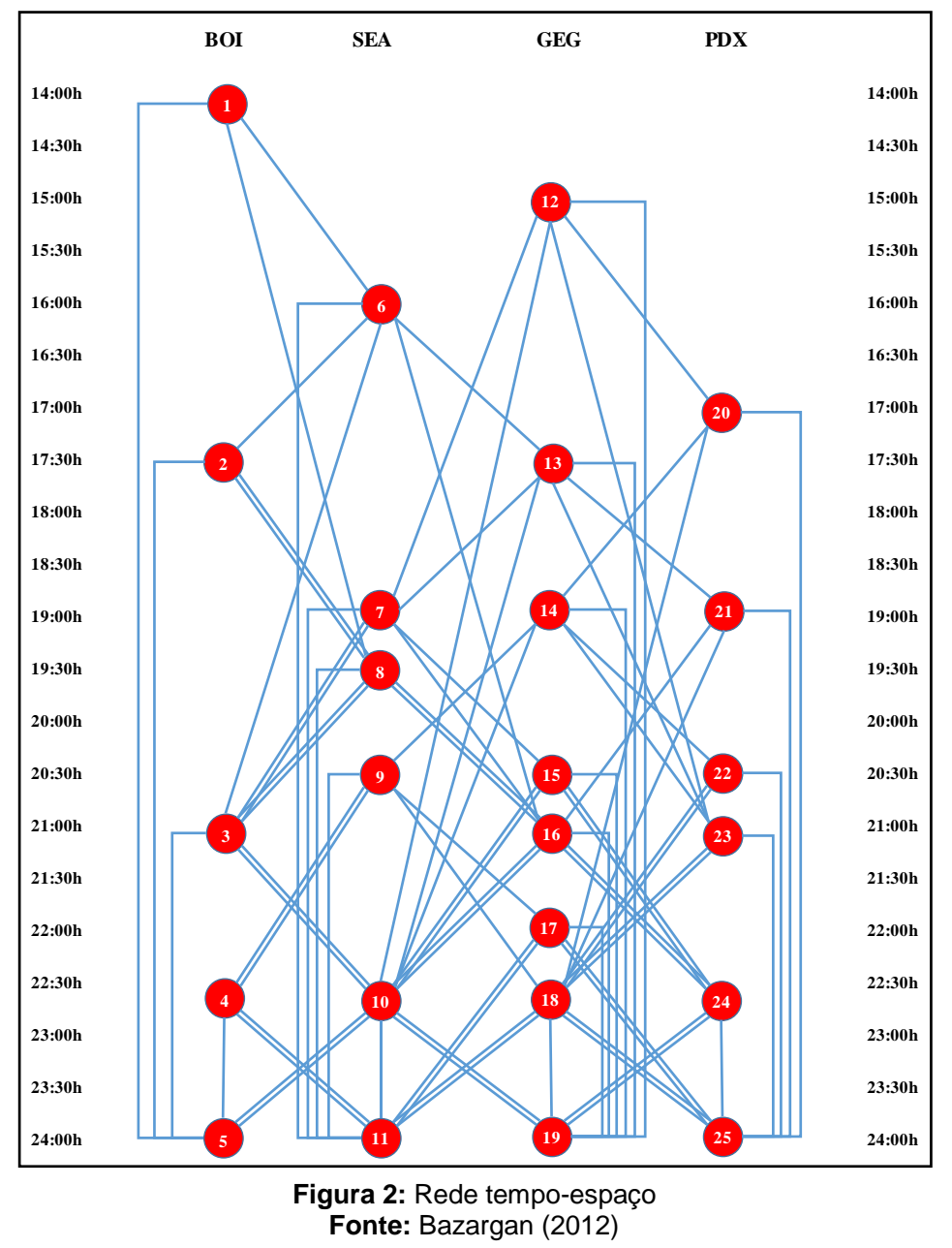

A proposta de solução do ARP consiste em determinar o novo sequenciamento das aeronaves de uma companhia aérea após a ocorrência de indisponibilidade de alguma(s) aeronave(s) em algum momento do dia, com os voos ocorrendo pontualmente ou com atrasos, e que resulte num menor custo total. Após a resolução do ARP, os atrasos dos voos podem ser por motivos inevitáveis, devido à indisponibilidade de aeronaves, ou proposital, como forma de melhor adequar a programação dos voos.

Dentro da abordagem do ARP, a variável de decisão $x_{i j}^{k}$ representa quais voos $k$ serão realizados entre os nós $i$ e $j$, mesmo que atrasados, $y_{k}$ significa quais voos $k$ serão cancelados e $z_{i}$ fornece a informação da quantidade de aeronaves nos nós terminais $i$ como forma de conservar as operações no dia seguinte.

A formulação do ARP, segundo método adotado por Arguello et al. (1998b) e por Bard et al. (2001), consiste na construção de uma rede tempo-espaço a partir do HOTRAN de uma empresa aérea e da modelagem matemática análoga ao problema do fluxo de custo mínimo.

A rede tempo-espaço é uma representação gráfica da localização temporal e espacial de todas as aeronaves disponíveis de uma companhia aérea e todas as possibilidades de realização dos voos programados sem atraso ou com atraso. Essa rede é construída a partir dos horários de pousos e decolagens programados, aeroportos a serem operados, tempo mínimo necessário em solo e horário limite para pousos e decolagens nos aeroportos. Como forma de exem- plificação, a Figura 2 representa a rede tempo-espaço construída a partir das informações contidas na Tabela 1.

Tabela 1. Exemplo de programação de voos Fonte: Bazargan (2012)

\begin{tabular}{cccccc}
\hline Aeronave & Voo & Origem & Destino & Partida & Chegada \\
\hline \multirow{2}{*}{1} & 11 & BOI & SEA & $14: 10$ & $15: 20$ \\
& 12 & SEA & GEG & $16: 05$ & $17: 00$ \\
& 13 & GEG & SEA & $17: 40$ & $18: 40$ \\
& 14 & SEA & BOI & $19: 20$ & $20: 35$ \\
\hline \multirow{2}{*}{2} & 21 & SEA & BOI & $15: 45$ & $17: 00$ \\
& 22 & BOI & SEA & $17: 40$ & $18: 50$ \\
& 23 & SEA & GEG & $19: 30$ & $20: 30$ \\
& 24 & GEG & SEA & $21: 15$ & $22: 15$ \\
\hline \multirow{2}{*}{3} & 31 & GEG & PDX & $15: 15$ & $16: 20$ \\
& 32 & PDX & GEG & $17: 30$ & $18: 30$ \\
& 33 & GEG & PDX & $19: 10$ & $20: 20$ \\
& 34 & PDX & GEG & $21: 00$ & $21: 55$ \\
\hline
\end{tabular}

Os elementos da rede são assim definidos a partir da ilustração apresentada na Figura 2:

- Eixo horizontal: conjunto de todos os aeroportos operados pela companhia aérea;

- Eixo vertical: discretização do tempo em bandas fixas. Os eventos de pousos e/ou decolagens nos aeroportos foram avaliados a cada 30 minutos;

- Nó: interseção entre um instante de tempo e a localização que podem ocorrer os eventos de pousos e/ou decolagens. A rede tempo-espaço é formada pelos conjuntos $i \in I$ e $j \in J$;

- Nós iniciais: representam os nós $i \in I$ onde ocorrem o primeiro evento de decolagem ou de pouso em cada aeroporto. No exemplo apresentado, os nós 1, 6 e 12 
suprem inicialmente a rede com as aeronaves que irão cumprir os voos programados;

- Nós terminais: representam os nós $j \in J$ onde ocorrem os últimos eventos de pouso em cada aeroporto. Os nós 5, 11 e 19 demandam aeronaves para darem continuidade aos voos programados no dia seguinte;

- Nós intermediários: são os nós $i \in I$ diferentes dos nós iniciais e dos nós terminais;

- Arcos terminais: conexões entre os nós iniciais/intermediários e os nós terminais e representam as possibilidades de uma dada aeronave localizada em $i \in I$ ser mantida no mesmo aeroporto como forma de suprir as operações no dia seguinte;

- Arcos de voos: conexões entre os diferentes nós que representam as possibilidades de realização de voos entre os nós $i$ e $j$ no horário ou com atraso;

- Conservação de fluxo: a partir do exemplo apresentado, os nós 1, 6 e 12 suprem aeronaves para o cumprimento dos voos programados e assim $a_{i}=1$. Os nós 5, 11 e 19 demandam, cada um deles, de uma aeronave, $h_{i}=1$, para que no dia seguinte as aeronaves possam dar início às operações. O restante dos nós conservam $a_{i}=0$. são:

As principais considerações sobre a abordagem adotada

- Para uma mesma aeronave, deve ser respeitado o tempo mínimo de 20 min em solo entre um pouso e uma decolagem subsequente para o desembarque e embarque de passageiros, descarregamento e carregamento de cargas, troca de tripulantes, abastecimento, limpeza e inspeções de manutenção;

- O período de recuperação das operações se estende até às $23: 45 \mathrm{~h}$;

- Não está sendo limitado o número máximo de pousos e decolagens por aeronave;

- Não está sendo levado em conta o planejamento da manutenção das aeronaves. Assim, caso seja necessário, considera-se que qualquer aeroporto poderá atender as demandas de manutenção de qualquer aeronave da frota;

- Não está sendo considerada a realização de voos de translados (sem passageiros);

- Os custos referentes aos atrasos e cancelamentos dos voos são estimativas;

- Considera-se que as aeronaves empregadas podem substituírem umas às outras;

- Não são considerados os problemas de alocação de tripulantes e passageiros; e

- Está sendo considerado o cumprimento da Instrução de Aviação Civil (IAC) no 1224 em que prevê o cancelamento eventual de voos e trocas de aeronaves desde que nenhum passageiro seja prejudicado (BRASIL, 2000c).

A seguir, é apresentado o modelo matemático definido a partir da construção da rede tempo-espaço para a resolução do ARP via PLI. Esse modelo baseia-se no problema de fluxo de custo mínimo.

\section{Índices}

$i, j:$ Índices de nó, e $k$ : Índice de voo.

Conjuntos

$F$ : Conjunto dos voos; $I$ : Conjunto dos nós que originam um voo $k ; J$ : Conjunto dos nós destinos de um voo $k$;

$P_{k}$ : Conjunto dos nós originários do voo $k ; H_{k}^{i}$ : Conjunto dos nós destinos do voo $k$ originário do nó $i \in I$;

$G_{i}$ : Conjunto dos voos originários do nó $i \in I ; L_{i}$ : Conjunto dos voos que terminam no nó $i \in J$;

$M_{k}^{i}$ : Conjunto dos nós de origens do voo $k$ que terminam no nó terminal $i \in J$; e

$Q_{i}$ : Conjunto dos nós que contêm arcos de suprimento para os nós terminais $i \in J$.

Dados

$a_{i}$ : Número de aeronaves disponível no nó $i \in I$, $c_{k}$ : Custo do cancelamento do voo $k$;

$d_{i j}^{k}$ : Custo do atraso do voo $k$ do nó $i$ para o nó $j$; e $h_{i}$; Número de aeronaves requeridas no nó terminal $i \in J$.

Variáveis

$x_{i j}^{k}=1$, se o voo $k \in F$ ocorrer entre os nós $i$ e $j$.

0 , se caso contrário

$y_{k}=1$, se o voo $k \in F$ é cancelado

0 , se caso contrário

$z_{i}$ : Número de aeronaves nos nós terminais.

Função Objetivo:

$$
\text { Minimizar } \sum_{k \in F} \sum_{i \in P_{k}} \sum_{j \in H_{k}^{i}} d_{i j}^{k} x_{i j}^{k}+\sum_{k \in F} c_{k} y_{k}
$$

Sujeito a:

$$
\begin{gathered}
\sum_{i \in P_{k}} \sum_{j \in H_{k}^{i}} x_{i j}^{k}+y_{k}=1, \quad \forall k \in F \\
\sum_{k \in G_{i}} \sum_{j \in H_{k}^{i}} x_{i j}^{k}+z_{i}-\sum_{k \in L_{i}} \sum_{j \in M_{k}^{i}} x_{i j}^{k}=a_{i}, \quad \forall i \in I \\
\sum_{k \in L_{i}} \sum_{j \in M_{k}^{i}} x_{i j}^{k}+\sum_{j \in Q_{i}} z_{j}=h_{i}, \quad \forall i \in J \\
x_{i j}^{k} \in\{0,1\} \quad \forall k \in F, i \in I e j \in H_{k}^{i} \\
y_{k} \in\{0,1\} \quad \forall k \in F \\
z_{i} \in Z^{+} \quad \forall i \in I
\end{gathered}
$$


DIAS, G.K.G.; SUBRAMANIAN, A.; CABRAL, L.A.F.

Tabela 2. Sequenciamento original das aeronaves segundo o HOTRAN da PASSAREDO

\begin{tabular}{|c|c|c|c|c|c|c|c|c|c|c|c|}
\hline Aeronave & $\begin{array}{c}N^{o} \text { do } \\
\text { voo }\end{array}$ & $\begin{array}{l}\text { Aeroporto } \\
\text { de origem }\end{array}$ & $\begin{array}{l}\text { Aeroporto } \\
\text { de destino }\end{array}$ & Partida & Chegada & Aeronave & $\begin{array}{c}\mathrm{N}^{\mathrm{o}} \text { do } \\
\text { voo }\end{array}$ & $\begin{array}{l}\text { Aeroporto } \\
\text { de origem }\end{array}$ & $\begin{array}{l}\text { Aeroporto } \\
\text { de destino }\end{array}$ & Partida & Chegada \\
\hline \multirow{8}{*}{1} & 2228 & SBSV & SBQV & $0: 05$ & $1: 15$ & \multirow{8}{*}{5} & 23162 & SBRP & SBGO & $9: 38$ & $10: 58$ \\
\hline & 2229 & SBQV & SBSV & $6: 00$ & $6: 58$ & & 23163 & SBGO & SBPJ & $11: 20$ & 13:08 \\
\hline & 22231 & SBSV & SBQV & $7: 30$ & $8: 35$ & & 23164 & SBPJ & SWGN & $13: 37$ & $14: 36$ \\
\hline & 22232 & SBQV & SBGR & $8: 55$ & $12: 00$ & & 23171 & SWGN & SBPJ & $14: 59$ & $15: 58$ \\
\hline & 2344 & SBGR & SBCA & $12: 20$ & $14: 30$ & & 23172 & SBPJ & SBGO & $16: 20$ & $18: 20$ \\
\hline & 2345 & SBCA & SBGR & $15: 00$ & $17: 15$ & & 23173 & SBGO & SBRP & $18: 51$ & 20:05 \\
\hline & 22241 & SBGR & SBQV & $18: 55$ & $21: 45$ & & 23174 & SBRP & SBGR & $20: 30$ & $21: 40$ \\
\hline & 22242 & SBQV & SBSV & 22:05 & 23:06 & & 22601 & SBGR & SBRP & $22: 00$ & 23:00 \\
\hline \multirow{8}{*}{2} & 22314 & SBBR & SBRP & $0: 10$ & $1: 50$ & \multirow{8}{*}{6} & 23461 & SBGR & SBCA & $0: 05$ & $1: 59$ \\
\hline & 22301 & SBRP & SBBR & $6: 40$ & $8: 24$ & & 23462 & SBCA & SBDO & $2: 20$ & $3: 20$ \\
\hline & 22302 & SBBR & SNBR & 9:07 & $10: 35$ & & 23471 & SBDO & SBCA & 4:00 & 5:00 \\
\hline & 22303 & SNBR & SBSV & $10: 55$ & $12: 35$ & & 23472 & SBCA & SBGR & $5: 20$ & $7: 30$ \\
\hline & 22304 & SBSV & SBQV & $13: 52$ & $15: 06$ & & 23603 & SBGR & SBTG & $15: 55$ & $12: 55$ \\
\hline & 22311 & SBQV & SBSV & $15: 26$ & $16: 36$ & & 23604 & SBTG & SBDO & $13: 15$ & $14: 15$ \\
\hline & 22312 & SBSV & SNBR & $17: 46$ & $19: 46$ & & 23611 & SBDO & SBTG & $18: 15$ & $19: 15$ \\
\hline & 22313 & SNBR & SBBR & $20: 11$ & $21: 41$ & & 23612 & SBTG & SBGR & $19: 45$ & $21: 35$ \\
\hline \multirow{10}{*}{3} & 2204 & SBRP & SBSR & $0: 22$ & $0: 55$ & \multirow{4}{*}{7} & 2340 & SBRP & SBUR & $0: 29$ & $1: 10$ \\
\hline & 2205 & SBSR & SBRP & 5:00 & $5: 40$ & & 2341 & SBUR & SBRP & 5:00 & $5: 43$ \\
\hline & 2254 & SBRP & SBGL & $6: 15$ & $7: 47$ & & 2267 & SBRP & SBGR & $9: 26$ & $10: 25$ \\
\hline & 2253 & SBGL & SBRP & $8: 22$ & $10: 05$ & & 2266 & SBGR & SBRP & $17: 50$ & $18: 50$ \\
\hline & 23781 & SBRP & SBBR & $10: 32$ & $12: 10$ & \multirow{8}{*}{8} & 23771 & SBUL & SBUR & $6: 20$ & $6: 50$ \\
\hline & 23782 & SBBR & SWSI & $12: 43$ & $15: 13$ & & 23772 & SBUR & SBGR & $7: 20$ & $8: 45$ \\
\hline & 22561 & SWSI & SBCY & $15: 35$ & $16: 21$ & & 2264 & SBGR & SBRP & $12: 30$ & $13: 32$ \\
\hline & 22562 & SBCY & SBGO & $16: 57$ & $19: 15$ & & 2258 & SBRP & SBGL & $14: 00$ & $15: 31$ \\
\hline & 22563 & SBGO & SBUL & $19: 47$ & $20: 45$ & & 2259 & SBGL & SBRP & $16: 04$ & $17: 40$ \\
\hline & 22564 & SBUL & SBRP & 21:05 & $21: 45$ & & 2269 & SBRP & SBGR & $18: 10$ & $19: 10$ \\
\hline \multirow{6}{*}{4} & 2271 & SBRP & SBBH & $6: 03$ & $7: 15$ & & 23721 & SBGR & SBUR & $19: 45$ & $21: 10$ \\
\hline & 2270 & SBBH & SBRP & $7: 40$ & 9:01 & & 23722 & SBUR & SBUL & $21: 30$ & $21: 58$ \\
\hline & 2273 & SBRP & SBBH & $14: 03$ & $15: 10$ & \multirow{8}{*}{9} & 22551 & SBRP & SBUL & $7: 15$ & $8: 00$ \\
\hline & 2272 & SBBH & SBRP & $15: 40$ & $16: 50$ & & 22552 & SBUL & SBGO & $8: 20$ & 9:05 \\
\hline & 2279 & SBRP & SBBH & $20: 25$ & $21: 32$ & & 22553 & SBGO & SBCY & $9: 43$ & $11: 27$ \\
\hline & 2278 & SBBH & SBRP & $21: 57$ & 23:08 & & 22554 & SBCY & SWSI & $11: 57$ & $13: 10$ \\
\hline \multirow{4}{*}{5} & 22602 & SBRP & SBTG & 0:02 & 1:00 & & 23791 & SWSI & SBBR & $14: 00$ & $16: 36$ \\
\hline & 22611 & SBTG & SBRP & $4: 30$ & $5: 35$ & & 23792 & SBBR & SBRP & $17: 15$ & $18: 55$ \\
\hline & 22612 & SBRP & SBGR & $6: 00$ & $7: 15$ & & 2252 & SBRP & SBGL & $19: 17$ & $20: 56$ \\
\hline & 23161 & SBGR & SBRP & 8:00 & 9:04 & & 2257 & SBGL & SBRP & $21: 28$ & 23:05 \\
\hline
\end{tabular}

A expressão (4) representa a função objetivo do modelo proposto. O primeiro termo significa o somatório dos custos devido ao atraso dos voos $k$ entre os nós $i$ e $j$. O segundo termo refere-se ao somatório dos custos devido ao cancelamento dos voos $k$.

As expressões (5) à (10) representam as restrições do modelo. As restrições (5) garantem que um determinado voo $k$ ou é cancelado ou é realizado entre os nós $i$ e $j$. As restrições (6) estabelecem o balanço de aeronaves no conjunto $I$. As restrições (7) garantem o balanço de aeronaves no conjunto $J$. As restrições (8), (9) e (10) são o domínio das variáveis de decisão.

\section{ASPECTOS METODOLÓGICOS}

Foi selecionado o HOTRAN da companhia aérea brasileira PASSAREDO, de domínio público disponibilizado no site da ANAC, para análise e discussão do ARP. Até consulta realizada em 29/01/2015, essa empresa é composta por 9 aeronaves, modelos ATR-72 que transporta até 70 passageiros, e opera 72 voos de segunda à sexta em 19 aeroportos brasileiros.

A metodologia para a resolução do ARP baseou-se em oito etapas, a saber: $1^{a}$ etapa: utilização do HOTRAN da PASSAREDO; $2^{a}$ etapa: sequenciamento original das aeronaves; $3^{a}$ etapa: construção da rede tempo-espaço; $4^{a}$ etapa: definição dos conjuntos do modelo matemático; $5^{a}$ etapa: resolução do modelo em pleno suprimento de aeronaves; $\sigma^{a}$ etapa: sequenciamento alternativo das aeronaves; $7^{a}$ etapa: criação das instâncias; e $8^{a}$ etapa: comparação entre as soluções ótimas e as soluções triviais.

A primeira etapa consistiu na utilização do $\mathrm{HO}$ TRAN da PASSAREDO para análise do ARP considerando os voos realizados entre as segundas-feiras e as sextas-feiras. Alguns voos que ocorrem durante a semana não ocorrem durante o final de semana, ou vice-versa, ou ainda nem todos os voos são realizados todos os dias da semana.

A segunda etapa consistiu na determinação dos sequenciamentos das 9 aeronaves operadas pela companhia aérea, representados na Tabela 2, como forma de cumprimento dos 72 voos previamente estabelecidos no HOTRAN. Durante o dia de operação, cada aeronave é responsável pela execução de um conjunto de voos.

Não existe uma única maneira de sequenciar as aeronaves e os requisitos básicos são que todos os voos sejam cumpridos com a quantidade de aeronaves da frota, existam aeronaves disponíveis para iniciar as operações nos aeroportos de origem, existam aeronaves disponíveis no término do dia como forma de garantir a continuidade das operações no dia seguinte, uma nova decolagem é realizada após um pouso e as aeronaves devem respeitar o tempo mínimo em solo entre um pouso e uma decolagem seguinte.

A terceira etapa consistiu na construção da rede tempo-espaço. Devido ao elevado número de nós e arcos de voos envolvidos, essa rede foi construída com o auxílio do Visual Basic for Applications (VBA) a partir dos sequenciamentos das aeronaves apresentadas na Tabela 2 e seguiu os passos básicos apresentados a seguir: 
$i, k=$ variáveis auxiliares; $\partial=$ conjunto dos diferentes aeroportos operados pela companhia aérea; $j=$ quantidade de aeroportos;

$\beta=$ tamanho da banda ( $\mathrm{min}) ; \delta=$ instantes de análise dos eventos incrementados do valor de $\beta$ (HH:mm);

$N=$ quantidade de aeronaves da frota; $\alpha=$ aeronave da frota; $\rho=$ aeroporto da primeira decolagem do dia da aeronave $\alpha$;

$\phi=$ horário da primeira decolagem do dia da aeronave $\alpha$ no aeroporto $\rho ; \sigma=$ aeroporto de pouso da aeronave $\alpha$;

$\eta$ = horário de pouso da aeronave $\alpha$ no aeroporto $\sigma ; \psi(\alpha, \eta, \sigma)=$ voos da aeronave $\alpha$, com pouso em $\sigma$ no horário $\eta$;

$\omega(\delta, \partial)=$ nós plotados em $\delta \cap \partial ; v=$ conjunto dos voos realizados pela frota segundo o HOTRAN;

$\gamma=$ quantidade de voos do conjunto $v ; \Omega=$ conjunto dos aeroportos de decolagem dos voos $v$;

$\mu=$ conjunto dos aeroportos de pouso dos voos $v ; d=$ tempo de duração dos voos $v$ entre os aeroportos $\Omega$ e $\mu$;

$t_{s}=$ tempo mínimo da aeronave em solo entre dois voos consecutivos; $t=$ horário dos nós plotados nas bandas $\delta$ da rede; e

$t^{\prime}=$ horário de chegada dos voos $v$ nos aeroportos $\mu$.
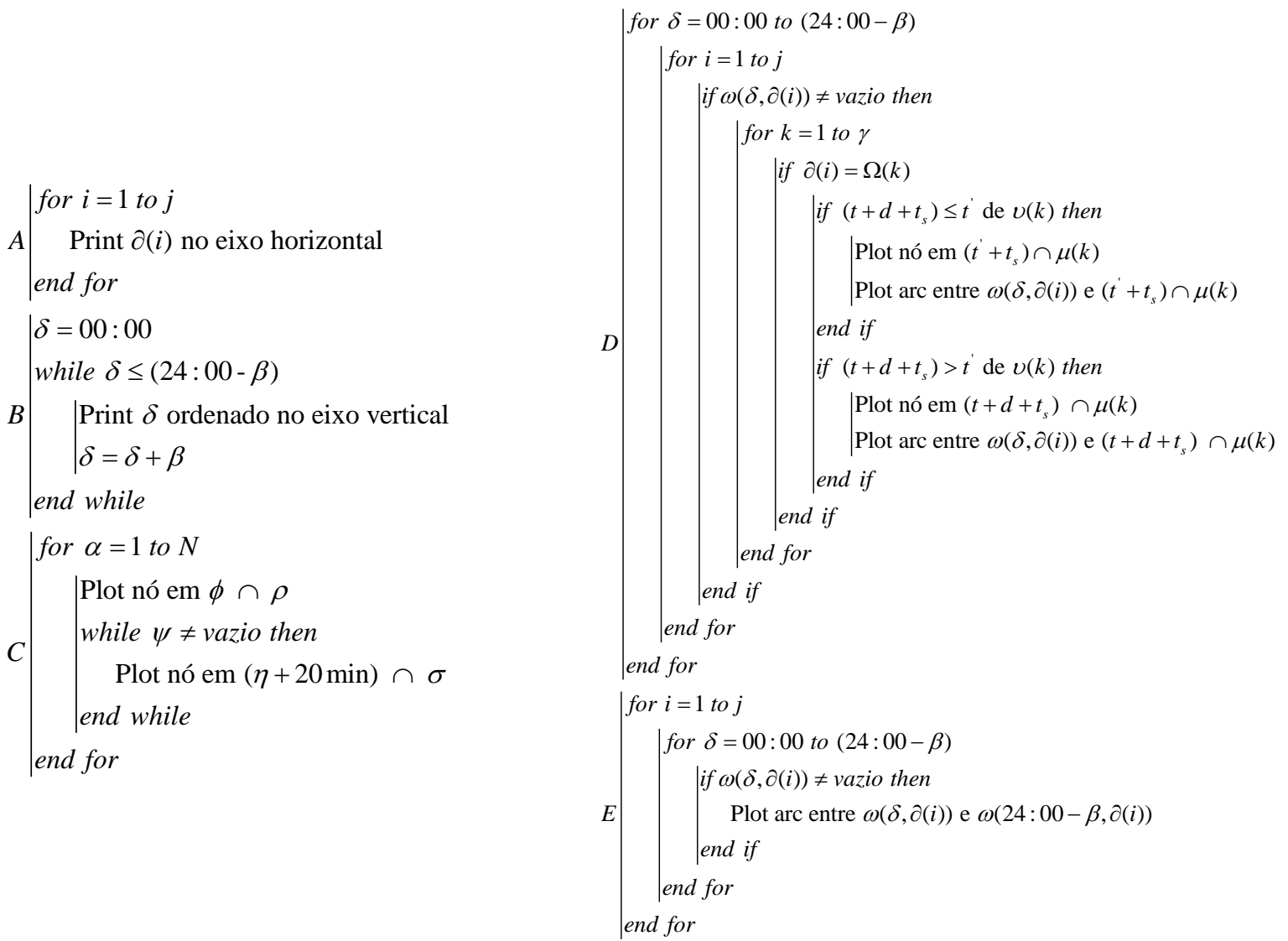

O passo A desse pseudocódigo representa a plotagem no eixo horizontal dos diferentes aeroportos operados pela companhia aérea. Para o caso particular da PASSAREDO, ela realiza voos em 19 diferentes aeroportos brasileiros.

$O$ passo $B$ representa a plotagem no eixo vertical os instantes dos eventos de pousos e decolagens das aeronaves e variam de 00:00h até (24:00h - $\beta$ ), onde $\beta$ representa o tamanho da banda.

$\mathrm{O}$ passo $C$ refere-se à plotagem dos nós iniciais da rede e representam o posicionamento e disponibilidade das aeronaves considerando o cumprimento dos voos estabelecidos na Tabela 2. Caso os valores de $\phi$ e $(\eta+20$ min) não coincidam com os valores de $\delta$, estes devem ser marcados no valor de $\delta$ imediatamente inferior.

O passo $D$ consiste na criação de novos nós e arcos a partir dos nós iniciais criados no passo $\mathrm{C}$ e no próprio passo D. Esses nós, em conexão com aqueles nós que o originaram, representam alternativas de realizações dos voos, mesmo que com atrasos.
Por fim, o passo $E$ representa a plotagem, num mesmo aeroporto, dos arcos entre os nós iniciais/intermediários e os nós terminais da rede. Esses arcos significam a possibilidade de conservar uma aeronave até o nó terminal para dar início às operações no dia seguinte.

A quarta etapa consistiu na extração dos conjuntos apresentados na formulação matemática a partir da construção da rede tempo-espaço para diferentes tamanhos de banda. Foram definidos o conjunto dos voos $F$ e seus subconjuntos $G_{i}$ e $L_{i}$, os quais podem ser realizados a partir do conjunto dos nós de origem $P_{k}$ com destino ao conjunto dos nós $H_{k}^{i}$.

Os voos $F$ podem ser realizados com custo nulo por atraso ou com algum custo por atraso, a depender da diferença de tempo entre o horário de chegada real e o horário de chegada planejado. 
DIAS, G.K.G.; SUBRAMANIAN, A.; CABRAL, L.A.F.

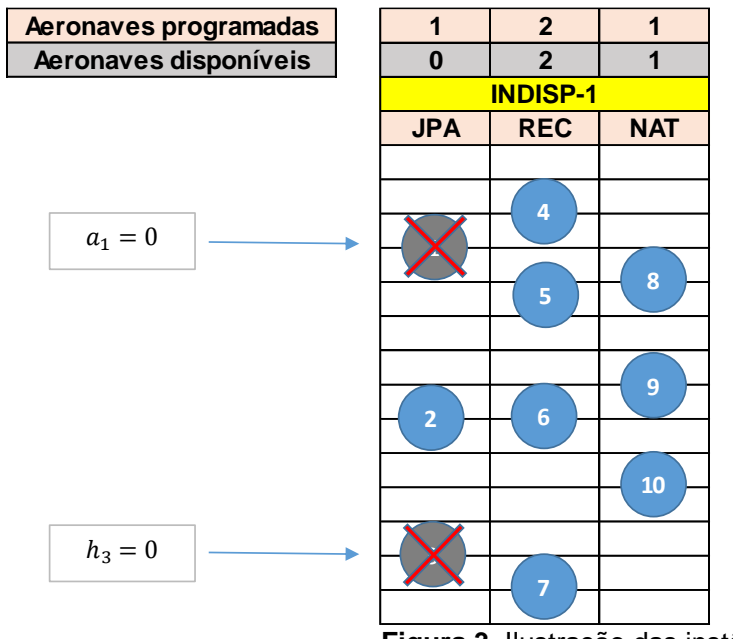

Figura 3. Ilustração das instâncias do tipo INDISP-X-Y-Z

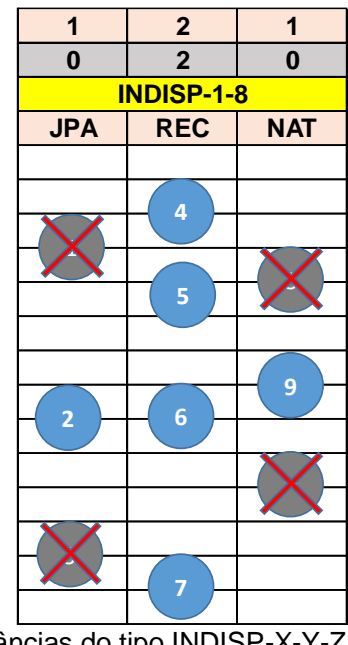

etapa poderá resultar em sequenciamentos das aeronaves idênticos aos já originalmente estabelecidos.

A criação das instâncias representa a sétima etapa e viabiliza o cumprimento da oitava etapa. Após o conhecimento dos nós da rede para os diferentes tamanhos de bandas, cada instância consiste nas variações dos valores de $a_{i}$

e $h_{i}$ nesses nós. Essas alterações simulam interrupções das operações ao modificar as quantidades de aeronaves supridas e demandadas, bem como as suas localizações nos diferentes nós das redes.

Para cada tamanho de banda empregado na construção da rede tempo-espaço, que gera um conjunto diferente de nós, foi convencionada a criação de 100 instâncias distintas que representam a indisponibilidade de até 3 aeronaves durante todo o dia de operação ou a indisponibilidade de até 3 aeronaves durante parte do dia de operação.

As instâncias de indisponibilidade de até 3 aeronaves durante todo o dia de operação são de três tipos: INDISP$\mathrm{X}$, INDISP-X-Y e INDISP-X-Y-Z. O valor de X representa o nó de suprimento que uma aeronave estará indisponível para operações, Y o local de indisponibilidade de uma segunda aeronave e $\mathrm{Z}$ de uma terceira aeronave. A Figura 3 representa esquematicamente um exemplo hipotético das instâncias INDISP-1, INDISP-1-8 e INDISP-1-8-4. A indisponibilidade de uma aeronave durante todo o dia de operação significa que os valores nos nós de suprimento $\left(a_{i}\right) \mathrm{e}$ de demanda $\left(h_{i}\right)$ são nulos.

As instâncias de disponibilidade de até 3 aeronaves durante parte do dia de operação são de três tipos: DISP-X, DISP-X-Y e DISP-X-Y-Z. O valor de X representa o nó de suprimento a partir do qual uma aeronave estará disponível para operações, Y o local a partir do qual uma segunda aeronave estará disponível e $\mathrm{Z}$ de uma terceira aeronave. Neste caso, a indisponibilidade parcial de uma aeronave significa que os valores nos nós de suprimento $\left(a_{i}\right)$ são nulos até o momento em que a aeronave volte a estar disponível para operação.

A oitava etapa consistiu na comparação entre os valores das soluções triviais para as diferentes instâncias, a partir do sequenciamento de cada aeronave, e os valores ótimos da função objetivo para essas mesmas instâncias. Esse critério de análise é importante para se ter a ideia de quão melhores são as soluções otimizadas em relação às soluções terminar por quais nós cada uma das 9 aeronaves devem percorrer para que todos os voos sejam cumpridos no horário e com uma função objetivo nula. Vale salientar que esta 

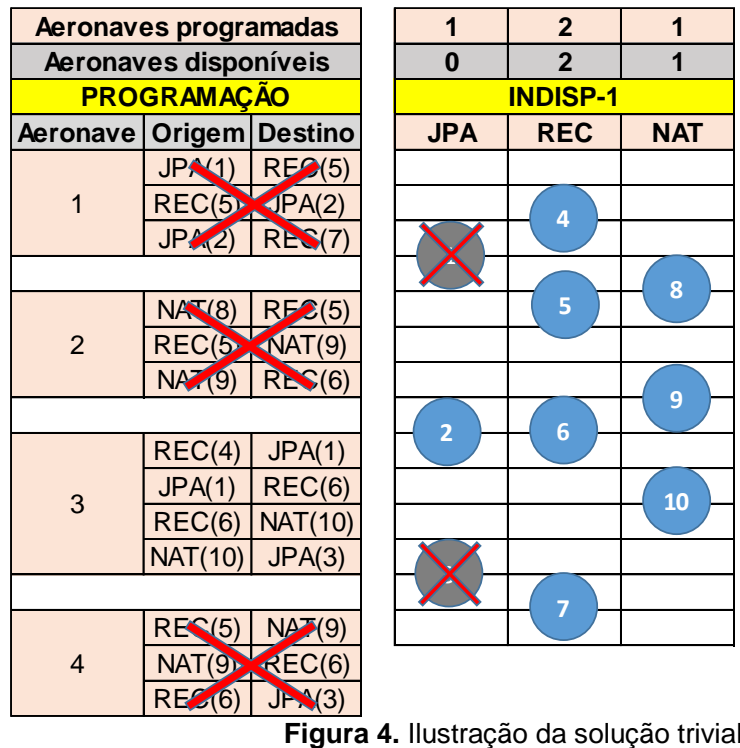
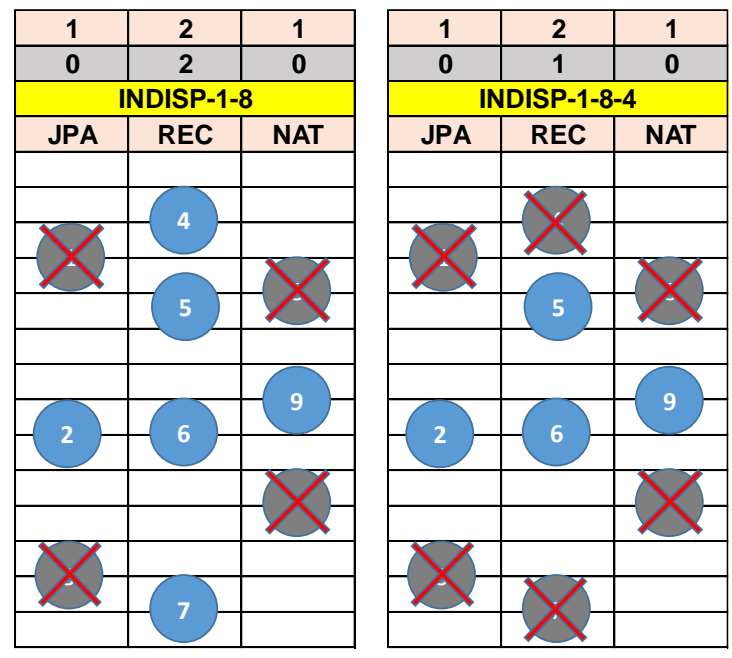

Figura 4. Ilustração da solução trivial para instâncias do tipo INDISP-X-Y-Z

convencionais (ou triviais) e já foi abordado por Bard et al. (2001) e Arguello et al. (1998b).

Para o caso das instâncias do tipo INDISP-X-Y-Z, a solução trivial é aquela considerando a soma dos custos por cancelamento dos voos que seriam realizados pelas aeronaves que estejam indisponíveis a partir do nó inicial de suprimento. A Figura 4 representa o que seriam as soluções triviais para as instâncias INDISP-1, INDISP-1-8 e INDISP-1-8-4. Neste caso, seriam cancelados todos os voos atribuídos às aeronaves $1,2 \mathrm{e} 4$, respectivamente.

Já para as instâncias do tipo DISP-X-Y-Z, a solução trivial é aquela considerando a soma dos custos por cancelamento dos voos que seriam realizados pelas aeronaves até os nós que as aeronaves passem a estar disponíveis.

A resolução do ARP envolvendo as diferentes instâncias eventualmente alteram o sequenciamento das aeronaves e fornecem o valor mínimo da função objetivo, em termos de custos, e também as variáveis de decisão $x_{i j}^{k}, y_{k} \mathrm{e}$ $z_{i}$. A solução informa quais voos $k$ devem ocorrer entre os nós $i$ e $j$, com atrasos ou pontuais, quais voos $k$ devem ser cancelados e quantas aeronaves $z_{i}$ estarão nos nós terminais para dar continuidade às operações no dia seguinte.

As principais características, limitações e contribuições do método podem ser assim relacionadas:

- É possível o acoplamento do ARP com outros subproblemas relevantes;

- Possibilita uma solução exata considerando a discretização do tempo em bandas fixas. Neste caso, diminuindo demais as bandas de tempo, por limitações de processamento computacional, torna-se improvável a resolução do problema via PLI;

- É possível a generalização da resolução, sem prejuízo da técnica, a partir dos dados do HOTRAN - ou equivalente no exterior - ou o emprego de dados em tempo real. Também é improvável a possibilidade de resolução do ARP via PLI para grandes malhas aéreas;

- O uso de instâncias e a comparação das soluções ótimas com as soluções triviais possibilita analisar a eficácia do modelo matemático e a técnica de otimização empregados; e
- A solução do ARP com aeronave extra pode auxiliar na análise de viabilidade econômica sobre a aquisição de mais uma aeronave na frota de uma companhia aérea.

\section{RESULTADOS}

Nesta seção são discutidas as diferentes configurações da rede tempo-espaço; o sequenciamento normal das aeronaves sem atrasos ou cancelamentos dos voos; e o comparativo entre as soluções ótimas encontradas e as soluções triviais para instâncias que simulam a indisponibilidade de aeronaves.

Para a resolução do ARP foram empregados o UFFLP versão 3.3.0, o qual consiste numa biblioteca de funções integrada ao solver CPLEX versão 12.5.1 e o VBA. Foi utilizado um computador com a plataforma Windows 7 e equipado com o processador i5 2,27 GHz.

Como a rede com banda de 15 min possui uma maior quantidade de nós e arcos de voos e, por representar uma maior complexidade de resolução, foi empregada para apresentação dos resultados obtidos.

A rede construída com banda de 15 min, e representada pela Figura 5, é composta por 96 bandas variando entre 00:00h e 23:45h, por 986 nós e por 3615 arcos de voos. Nessa figura, os traçados em destaque representam os sequenciamentos alternativos das 9 aeronaves disponíveis para o cumprimento dos voos sem atrasos previstos na Tabela 2. Vale salientar que, para não comprometer a clareza da leitura dessa figura, a representação gráfica dos arcos terminais foram omitidos, apesar destes terem sido considerados na resolução do modelo matemático.

Os sequenciamentos apresentados na Figura 5 foram feitos a partir da resolução do modelo matemático empregando todas as 9 aeronaves localizadas nos nós de suprimentos conforme apresentados na Tabela 2. Para o caso da rede construída com uma banda de 15 min, e conforme também ilustrados na Figura 5, os pares de nós de suprimento e de demanda por 1 aeronave foram 1-46 em SBSV, 84-146 em SBGR, 204-255 em SBBR, 256-324 em SBRP, 257-324 em SBRP, 257-324 em SBRP, 260-324 em SBRP, 261-324 em SBRP e 624-682 em SBUL. O valor da função objetivo, como esperado, foi nulo. 
DIAS, G.K.G.; SUBRAMANIAN, A.; CABRAL, L.A.F.

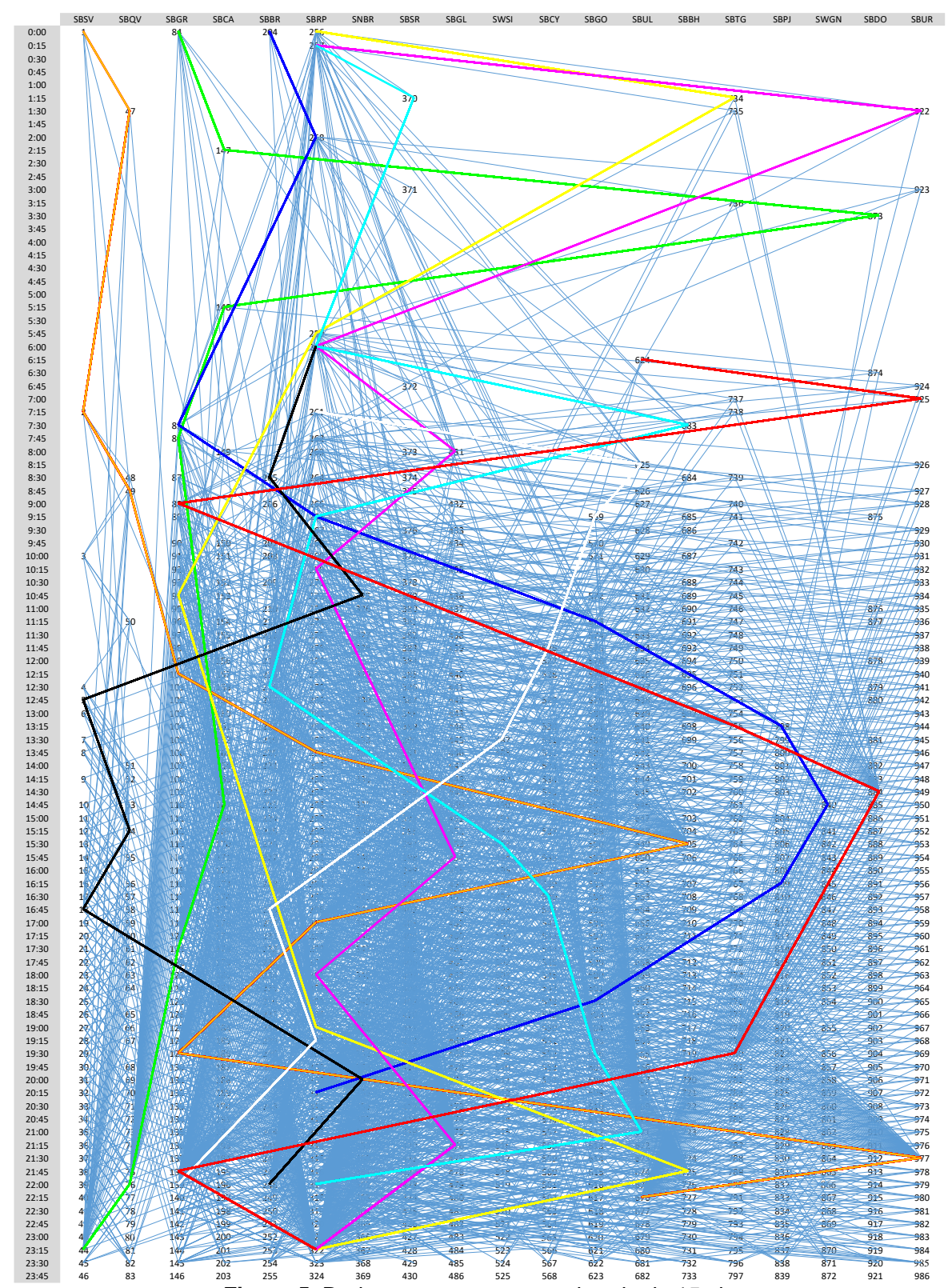

Figura 5. Rede tempo-espaço com banda de $15 \mathrm{~min}^{{ }^{203}}$

Os valores encontrados para $x_{i j}^{k}$, após a resolução do modelo nas condições descritas anteriormente, são apresentados graficamente na Figura 5 e definem os novos sequenciamentos das 9 aeronaves. Os locais de suprimento de 1 aeronave e de demanda por 1 aeronave são aqueles representados pelos pares de nós, respectivamente, 1-676, 84-44, 204-310, 256-322, 257-322, 257-317, 260-248, 261-139 е 624-322.

O ARP foi analisado a partir da geração de 100 instâncias tomando como base os nós de suprimento e demanda nos diferentes aeroportos e foram do tipo INDISP$X$, INDISP-X-Y, INDISP-X-Y-Z, DISP-X, DISP-X-Y e DISP-X-Y-Z.

O comparativo entre as soluções ótimas e as soluções triviais das instâncias testadas é apresentada na Figura 6. Para todas as situações, as soluções ótimas tiveram os seus valores inferiores ou pelo menos iguais às soluções triviais. Para uma rede tempo-espaço com banda de $15 \mathrm{~min}$, a diferença percentual média entre as soluções ótimas e as soluções triviais de todas as instâncias testadas foi de $41,15 \%$.

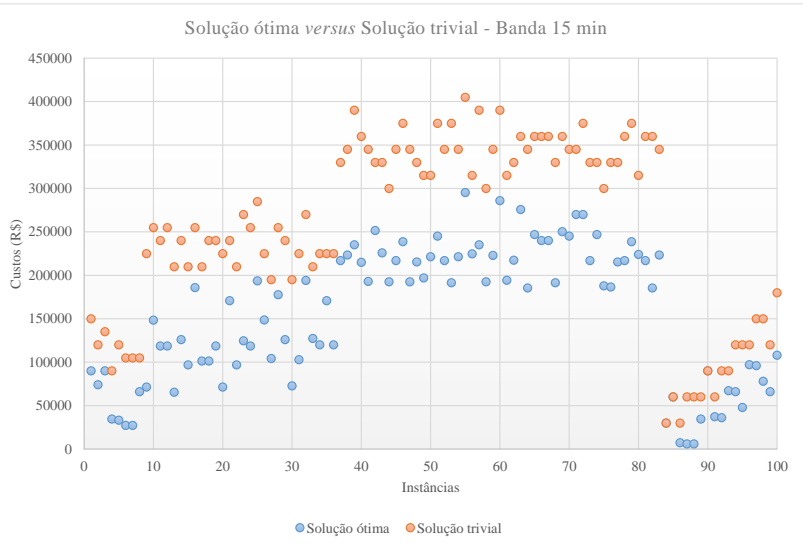

Figura 6. Comparativo entre as soluções

A resolução do ARP para uma rede com banda de 15 min e aeronave extra consistiu em disponibilizar uma aeronave a mais no nó 256, em SBRP, aeroporto com o maior número de voos realizados pela companhia aérea em estudo, para o uso em situações de indisponibilidade das outras aeronaves. 
Tabela 3. Comparativo entre as soluções

\begin{tabular}{|c|c|c|c|c|c|}
\hline Banda (min) & $\mathbf{N}^{\circ}$ bandas & Nós & Arcos & Diferença (\%) & Tempo (s) \\
\hline 30 & 48 & 532 & 1998 & 38,24 & 30 \\
\hline 20 & 72 & 768 & 2838 & 40,44 & 60 \\
\hline 15 & 96 & 986 & 3615 & 41,15 & 100 \\
\hline \multicolumn{6}{|c|}{ Rede tempo-espaco com banda de $15 \mathrm{~min}$ e aeronave extra } \\
\hline Banda (min) & $\mathbf{N}^{\circ}$ bandas & $\mathbf{N}^{\mathbf{o}}$ nós & $\mathbf{N}^{0} \operatorname{arcos}$ & Diferença (\%) & Tempo (s) \\
\hline 15 & 96 & 986 & 3615 & 38,68 & 100 \\
\hline
\end{tabular}

Das 100 instâncias que foram testadas para uma rede com banda de $15 \mathrm{~min}, 95$ delas também foram testadas para uma rede com banda de 15 min e aeronave extra. A diferença média percentual entre as soluções ótimas para uma rede com banda de 15 min com aeronave extra e sem aeronave extra foi de $38,68 \%$. Para todas os casos, as soluções ótimas com aeronave extra foram menores do que as soluções sem essa aeronave a mais.

Apesar de o emprego de uma aeronave extra aparentemente ser vantajoso, o real benefício não é conclusivo uma vez que os custos de aquisição e manutenção de uma aeronave a mais na frota são elevados.

A Tabela 3 apresenta dados comparativos entre os tamanhos de bandas de $30 \mathrm{~min}, 20 \mathrm{~min}$ e $15 \mathrm{~min}$ para a construção das redes tempo-espaço no que se referem ao número de bandas, de nós e de arcos, diferenças médias entre as soluções ótimas e as soluções triviais e o tempo de processamento médio para a resolução de uma instância. Na última linha é apresentada a diferença média entre as soluções ótimas para o caso da rede tempo-espaço com banda de $15 \mathrm{~min}$ sem aeronave extra e com aeronave extra.

É possível observar que quanto menor a banda de tempo empregada, melhor será a solução encontrada, tendo em vista que um número maior de arcos de voos será analisado, e maior será o tempo de processamento computacional. Entretanto, considerando uma determinada malha fixa de voos, existe um limite para a diminuição dessa banda de modo que seja possível a resolução do ARP via PLI.

\section{CONCLUSÃO}

Esse trabalho propôs uma solução para o ARP de companhias aéreas regulares de pequeno porte a partir dos dados da empresa PASSAREDO e da metodologia abordada por Arguello et al. (1998b) e por Bard et al. (2001).

A partir do percurso metodológico apresentado na seção 3, as soluções obtidas para o caso da rede construída a partir da banda com 15 min foram, em média, 41,15\% menores do que as soluções triviais para as 100 instâncias testadas. Isso mostra que é possível o desenvolvimento de ferramentas mais objetivas e que complementem a usual prática utilizada pelas companhias aéreas de resolver os problemas de interrupções das suas operações por meio da análise pontual, e não sistemática, de toda a malha aérea que ela opera.

Os resultados obtidos com o emprego de uma aeronave extra se mostraram atrativos. Para uma mesma rede tempo-espaço com banda de 15 min, das 95 instâncias testadas, em média as soluções com aeronave reserva foram $38,68 \%$ menores do que as soluções sem aeronave reserva. No entanto, esse aparente benefício não é conclusivo por ser necessária uma análise de viabilidade econômica mais detalhada por parte da companhia aérea sobre a aquisição e manutenção de um novo equipamento na frota.
Nem sempre os estudos atuais sobre o assunto são dedicados exclusivamente ao insumo aeronave e claros suficientemente para generalizações. Assim, a metodologia apresentada permite o emprego em outras companhias aéreas de pequeno porte, bem como a fácil comparação dos resultados obtidos com as soluções conceitualmente definidas como triviais. Além disso, devido à característica de resolução exata, essa metodologia pode ser empregada até o seu limite máximo de nós e arcos para a comparação com metaheurísticas desenvolvidas para a resolução de problemas maiores.

Trabalhos futuros podem empregar metaheurísticas para a resolução do ARP em situações que envolvam um elevado número de aeronaves, de voos e de aeroportos operados por uma companhia aérea brasileira.

Outros trabalhos podem ser desenvolvidos analisando a rede tempo-espaço não mais estática, ou seja, construída e analisada a partir de uma programação predefinida, mas reiteradamente reconstruída após a ocorrência não só de indisponibilidade de aeronaves durante um período de tempo, mas também devido ao atraso de aeronaves ao longo do dia de operação.

Pode-se analisar também a possibilidade de aprimoramento do cálculo dos custos por atraso e cancelamento dos voos e passar a considerar a realização de voos de translado de algumas aeronaves como forma de auxiliar na recuperação das operações após a ocorrência de interrupções. $\mathrm{O}$ voo de translado é entendido como aquele realizado sem passageiros a bordo e entre aeroportos que não necessariamente possuam voos previamente programados.

Por fim, o acoplamento do ARP com os problemas de recuperação de tripulantes e de passageiros e a otimização do planejamento da manutenção entre as bases de manutenção situadas nos aeroportos operados pela companhia aérea, representa um importante problema a ser tratado por relacionar alguns dos mais importantes insumos operacionais ao seu maior sentido de existir: os passageiros.

\section{REFERÊNCIAS}

ARGÜELLO, Michael F.; BARD, Jonathan F.; YU, Gang (1997a). A GRASP for aircraft routing in response to groundings and delays. Journal of Combinatorial Optimization, v. 1, n. 3, p. 211-228. DOI: 10.1023/A:1009772208981.

ARGÜELLO, Michael F.; BARD, Jonathan F.; YU, Gang (1998b). Models and methods for managing airline irregular operations. In: Operations Research in the Airline Industry. Springer US, p. 1-45. DOI: 10.1007/978-1-4615-5501-8_1.

ANAC (2013). Anuário do Transporte Aéreo: Dados Estatísticos e Econômicos de 2013. Brasília: Anac, 207 p.

BARD, Jonathan F.; YU, Gang; ARGUELLO, Michael F (2001). Optimizing aircraft routings in response to groundings and delays. IIE Transactions, v. 33, n. 10, p. 931-947. DOI: 10.1080/07408170108936885. 
BAZARGAN, Massoud (2012). Airline operations and scheduling. Ashgate Publishing, Ltd.

BISAILLON, Serge et al. (2011). A large neighbourhood search heuristic for the aircraft and passenger recovery problem. 4OR, v. 9 , n. 2, p. 139-157. DOI: 10.1007/s10288-010-0145-5.

BELOBABA, Peter; ODONI, Amedeo; BARNHART, Cynthia (2009). The global airline industry. John Wiley \& Sons. DOI: 10.1002/9780470744734.

BRASIL (1986a). Lei $n^{\circ} 7565,9$ de dezembro de 1986. Código Brasileiro de Aeronáutica. Brasília.

BRASIL (2000b). Agência Nacional de Aviação Civil. IAC 1223, 19 de janeiro de 2000. Normas para confecção e aprovação de HOTRAN. Rio de Janeiro.

BRASIL (2000c). Agência Nacional de Aviação Civil. IAC 1224, 19 de janeiro de 2000. Normas para alterações em voos regulares e realização de voos não-regulares. Rio de Janeiro.

COOK, A.; TANNER, G (2013). Standard Inputs for EUROCONTROL Cost Benefit Analyses. Department of Transport Studies University of Westminster, University of California, Riverside.

HANSEN, Mark; ZOU, Bo (2013). Airport Operational Performance and Its Impact on Airline Cost. Modelling and Managing Airport Performance, p. 119-143. DOI: 10.1002/9781118535844.ch5.

KOHL, Niklas et al. (2007). Airline disruption management perspectives, experiences and outlook. Journal of Air Transport Management, v. 13, n. 3, p. 149-162. DOI:

10.1016/j.jairtraman.2007.01.001.

NICKKAR, Amirreza et al. (2015). Developing a model for solving the flight perturbation problem. Journal of Airline and Airport Management, v. 5, n. 1, p. 18-38. DOI: 10.3926/jairm.31.

TAHA, Hamdy A. Pesquisa operacional: uma visão geral. 8. ed. São Paulo: Pearson Education do Brasil, 2008. 359 p.

TEODOROVIĆ, Dušan; GUBERINIĆ, Slobodan (1984). Optimal dispatching strategy on an airline network after a schedule perturbation. European Journal of Operational Research, v. 15, n. 2, p. 178-182.DOI: 10.1016/03772217(84)90207-8. 